\title{
Quantifying point-mutations in shotgun metagenomic data
}

4 Affiliations

$5{ }^{1}$ Wisconsin Institute of Discovery, University of Wisconsin-Madison, 330 North Orchard Street,

6 Madison WI 53715, USA

$7 \quad{ }^{2}$ Department of Biotechnology, School of Bioengineering, SRM Institute of Science and

8 Technology, Kattankulathur 603203, Tamil Nadu, India

$9{ }^{3}$ Chalmers Computational Systems Biology Infrastructure, Chalmers University of Technology,

10 SE-412 96, Gothenburg, Sweden

$11{ }^{4}$ Department of Infectious Diseases, Institute of Biomedicine, The Sahlgrenska Academy,

12 University of Gothenburg, Guldhedsgatan 10, SE-413 46, Gothenburg, Sweden

$13{ }^{5}$ Centre for Antibiotic Resistance research (CARe) at University of Gothenburg, Gothenburg,

14 Sweden

$15 *$ Corresponding author: Johan Bengtsson-Palme, Department of Infectious Diseases,

16 Guldhedsgatan 10, SE-413 46, Gothenburg, Sweden. Phone: +46 3134246 26. Fax: +46 3184

$17 \quad 61$ 13. E-mail address: johan.bengtsson-palme@microbiology.se 
20

21

\section{Abstract}

Metagenomics has emerged as a central technique for studying the structure and function of microbial communities. Often the functional analysis is restricted to classification into broad functional categories. However, important phenotypic differences, such as resistance to antibiotics, are often the result of just one or a few point mutations in otherwise identical sequences. Bioinformatic methods for metagenomic analysis have generally been poor at accounting for this fact, resulting in a somewhat limited picture of important aspects of microbial communities. Here, we address this problem by providing a software tool called Mumame, which can distinguish between wildtype and mutated sequences in shotgun metagenomic data and quantify their relative abundances. We demonstrate the utility of the tool by quantifying antibiotic resistance mutations in several publicly available metagenomic data sets. We also identified that sequencing depth is a key factor to detect rare mutations. Therefore, much larger numbers of sequences may be required for reliable detection of mutations than for most other applications of shotgun metagenomics. Mumame is freely available from http://microbiology.se/software/mumame

\section{Keywords}

Antibiotic resistance, Bioinformatic tools, Metagenomics, Mutation frequencies, Mutation mapping, Statistical methods 
40

\section{Introduction}

The revolution in sequencing capacity has created an unprecedented ability to glimpse into the functionality of microbial communities, using large-scale shotgun metagenomic techniques (Quince et al. 2017). This has yielded important insights into broad functional patterns of microbial consortia (Yooseph et al. 2007; Human Microbiome Project Consortium 2012; Sunagawa et al. 2015). However, while overall pathway abundances inferred from metagenomic data can tell us much about the general functions of communities and how they change with e.g. environmental gradients (Bengtsson-Palme 2018; Bahram et al. 2018), there are many important functional differences that are hidden in the subtleties of these communities (Österlund et al. 2017). For example, many antibiotic resistance phenotypes are the results of single point mutations rather than acquisition of novel pathways or genes (Johnning et al. 2013). This complicates the studies of selection pressures in environmental communities, as analysis of such mutations is generally limited to a narrow range of species (Johnning et al. 2015b; Johnning et al. 2015a; Kraupner et al. 2018).

Because of the immense increase in available sequence data, it would be desirable to study these mutations from shotgun metagenomic libraries, much as other traits have been studied at a large scale (Pal et al. 2016). However, attempts to quantify point mutations in metagenomic sequencing data often go wrong because the methods do not sufficiently well distinguish between mutated and wildtype variants of the same gene. For example, a sequenced read may map to a region identical in the mutated and wildtype variant of a gene, causing problems for quantifying their relative proportions (Bengtsson-Palme et al. 2017). In addition, because the sought-after mutations generally are rare in most types of sample, and metagenomic studies are often under- 
sampled in terms of replicates (Jonsson et al. 2016a), commonly applied statistics methods may not be sufficiently sensitive to reliably detect differences between samples (Jonsson et al. 2016b).

In this study, we attempt to provide a partial remedy to these problems through the introduction of a software tool - Mumame - that can quantify and distinguish between wildtype and mutated gene variants in metagenomic data, and through suggesting a statistical framework for handling the output data of the software. We further demonstrate the ability of the method to detect relevant differences between environmental sample types, estimate the sequencing depths required for the method to perform reliably through simulations, and exemplify the utility of the software on detecting resistance mutations in publicly available metagenomes. The Mumame software package is open-source and freely available from http://microbiology.se/software/mumame

\section{Methods}

\section{Software implementation}

Mumame is implemented in Perl and consists of two commands: mumame, which performs mapping to database of mutations, and mumame_build with builds the database for the former command. The mumame_build command takes a FASTA sequence file and a list of mutations (CSV format) as input. For each entry in the mutation list, it finds the corresponding sequence(s) in the FASTA file, either by sequence identifier or by CARD ARO accessions (Jia et al. 2016). It then excerpts a number of residues upstream and downstream of the mutation position (by default 20 residues for proteins and 55 for nucleotide sequences) and creates one wildtype version and one mutated version of the sequence excerpt with unique sequence IDs. For cases 
where multiple mutations can occur close to each other on the same sequence, the software attempts to create all possible combinations of mutations (if memory permits - is some situations this is not possible because the number of combinations increase exponentially). The software tool also generates a mapping file between sequence IDs in the database and mutation information from the list.

The main mumame command takes any number of input files containing DNA sequence reads in FASTA or FASTQ format and maps those against the Mumame database using Usearch (Edgar 2010). For this mapping, the software runs Usearch in search_global mode with target coverage set to 0.55 (by default; any value $\geq 0.51$ should be feasible for target coverage). The output is then mapped to the wildtype or mutation information in the Mumame database, and data is collected for each input file and combined into one single output table.

The output table generated by Mumame can then be analyzed using the R script ( $\mathrm{R}$ Core Team 2016) supplied with the Mumame package. The script reads the read counts for all mutation positions detected, both for wildtype and mutated sequences, and assesses if there are significantly different proportions of mutations between different sample groups directly through a generalized linear model. Alternatively, an overdispersed Poisson generalized linear model accounting for the discrete nature of the data and the differences in sequencing depth can be used (Jonsson et al. 2016b; Bengtsson-Palme et al. 2017). The Poisson model is preferable when the number of counts for a targeted gene is low in all sample groups.

\section{Quantification of mutations in metagenomes}

To quantify the abundances of fluoroquinolone resistance mutations in the gyr $A$ and $\operatorname{par} C$ genes (Johnning et al. 2015b), we downloaded the CARD database on 2018-05-24 (Jia et al. 2016). We 5 
extracted all mutation information regarding the $g y r A$ and $\operatorname{par} C$ genes from the "snps.txt" file and created a new file with that information. We then created a new Mumame database, with the following command: “mumame_build -i card-data/protein_fasta_protein_variant_model.fasta -m gyrA_parC_snps.txt -o gyrA_parC”. That database was used to map all the reads from the samples generated by Kraupner et al. (2018) to the database using Mumame in the Usearch mode

110 (Edgar 2010) and the following options “-d gyrA_parC -c 0.95". We did this both for the

111 shotgun metagenomics data as well as for the amplicon sequences derived specifically from

112 Enterobacteriaceae $g y r A$ and $\operatorname{par} C$ genes. Prior to this sequence mapping raw reads were quality

113 filtered using Trim Galore! (Babraham Bioinformatics 2012) with the settings "-e 0.1 -q 28 -O 1".

114 We then used the R script ( $\mathrm{R}$ Core Team 2016) provided with the Mumame software to compare

115 the matches to mutated and wildtype sequences in the database. The same database and method

116 combination was used to quantify fluoroquinolone resistance mutations in sequence data from an

117 Indian lake exposed to ciprofloxacin pollution (Bengtsson-Palme et al. 2014), as well as in an

118 Indian river upstream and downstream of a wastewater treatment plant processing

119 pharmaceutical waste (Kristiansson et al. 2011; Pal et al. 2016). These samples were preprocessed

120 in the same way as in the Indian lake study (Bengtsson-Palme et al. 2014).

121 To quantify resistance mutations to tetracycline in the sequence data generated by (Lundström et

122 al. 2016), we created a Mumame database for tetracycline resistance mutations in the 16S rRNA

123 gene. We extracted the mutational information related to tetracycline from the CARD "snps.txt"

124 file and then built the database using the following command: "mumame_build -i card-

125 data/nucleotide_fasta_rRNA_gene_variant_model.fasta -m Tet_snps.txt -o Tet -n”. We then

126 mapped all reads from the Lundström et al. (2016) data to the Mumame database using the 
options “-d Tet -c 0.95 -n”. Reads were quality filtered and statistical differences were assessed as

128 above.

\section{Software evaluation}

130 To assess the limitations of the method in terms of sequencing depth, the samples from the highest and lowest ciprofloxacin concentrations generated by Kraupner et al. (2018; $10 \mu \mathrm{g} / \mathrm{L}$ and sequencing depths and average effect sizes calculated for the significantly altered genes.

\section{Results}

Mumame can quantify point mutation frequencies in metagenomic data

138 As a proof-of-concept that our method to identify point mutations in metagenomic sequence

139 data is functional, we used Mumame to quantify the mutations in amplicon data from the the

140 gyr $A$ and $\operatorname{par} C$ genes. These genes are targets of fluoroquinolone antibiotics, and often acquire

141 resistance mutations attaining high levels of resistance. We quantified such mutations in an

142 amplicon data set specifically targeting these two genes in Escherichia coli. This data set derives

143 from an exposure study with increasing ciprofloxacin concentrations, and enrichments of

144 mutations in the classical fluoroquinolone resistance determining positions S83 and D87 (gyrA)

145 and S80 and E84 ( $\tan C$ ) have previously been verified using other bioinformatic methods

146 (Kraupner et al. 2018). This data set therefore serves as an ideal positive control for our novel

147 method. We found that Mumame were able to identify the difference between the highest 
148 concentration $(10 \mu \mathrm{g} / \mathrm{L})$ and the lower ones reported in the original study (Figure 1). However,

149 Mumame only reported an average frequency of mutations of around $11-12 \%$ for gyrA mutations

150 (Figure 1A), while the original paper finds frequencies of $60-85 \%$ (S83) and $30-40 \%$ (D87). The

151 A67 position was not quantified in the original paper. The reason for the discrepancies is

152 unknown, but it is likely caused by a taxonomic filtration step that selects for E. coli reads used in

153 the Kraupner et al. study, while Mumame does not perform prior filtering. The decision to

154 exclude filtering was made in order to mimic a situation with true metagenomic data where

155 several target species may co-exist. For parC, Mumame only quantified the S80 position (Figure

156 1C), because the E84 mutations were not included in the version of the CARD database used for

157 this study. For position S80, Mumame identified around 35\% mutated sequences at the highest

158 concentration of ciprofloxacin, while the original study reported around 50\%. 
Amplicon data

\section{A)}

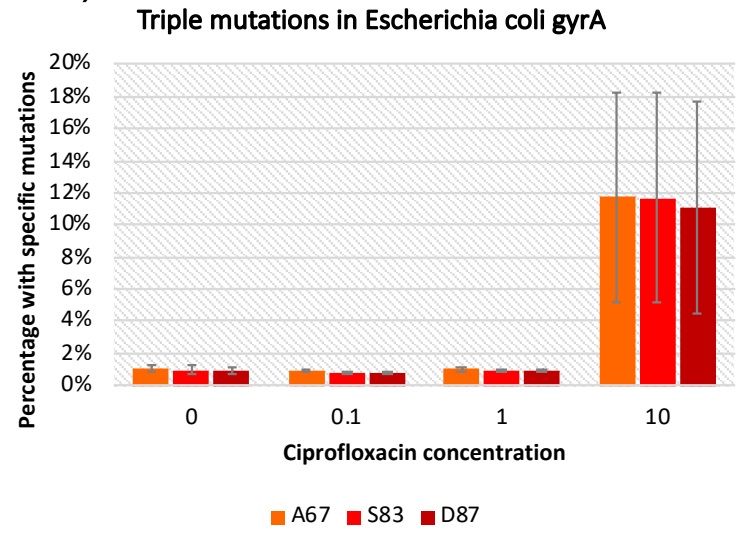

C)

Single mutation in Escherichia coli parC

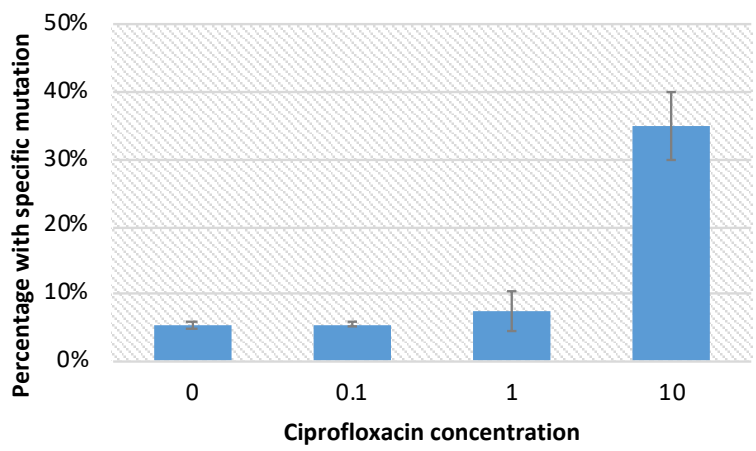

$\mathrm{S} 80$
Shotgun metagenomic data

B)

Triple mutations in Escherichia coli gyrA

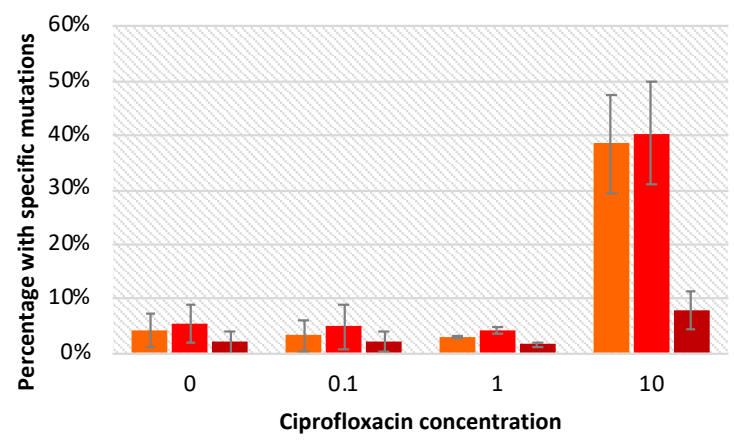

- A67 $\square$ S83 D D87

D)

Single mutation in Escherichia coli parC

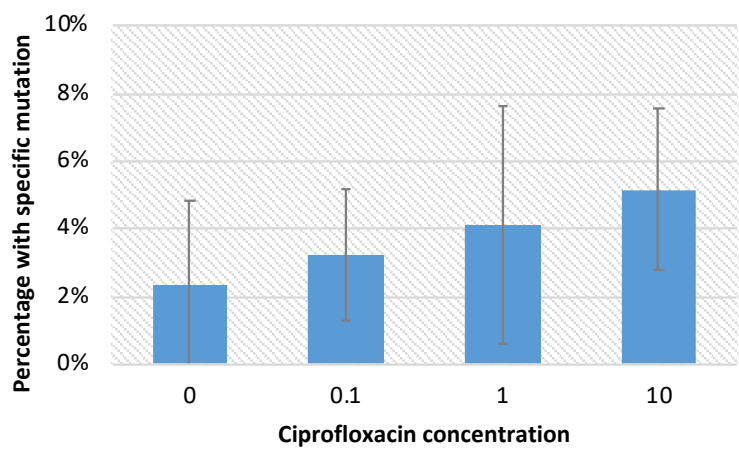

S80

Figure 1. Total mutation frequencies quantified using Mumame for three known mutations conferring resistance to fluoroquinolone in the E. coli gyrA gene based on amplicon sequencing (A) and shotgun metagenomic data (B) from the same samples. Corresponding data for the $\mathrm{S} 80$ mutation in parC is shown in (C) for amplicon data and (D) for shotgun data.

We next evaluated the performance of Mumame on the real shotgun data that was also generated

166 from the same samples as the amplicon libraries. Ideally, this analysis should generate virtually the

167 same result as the amplicon analysis. Indeed, we found similar results for the A67 and S83 gyr $A$

168 mutations (Figure 1B). For the D87 mutation, the frequencies were much lower than for the

169 other two mutations, albeit still significantly larger than at the lower concentrations $(\mathrm{p}<0.01)$. 
170 For the parC gene, the shotgun metagenomic analysis was too noisy to generate a statistically

171 significant result, which was highly surprising to us (Figure 1D). Taken together, these results

172 indicate the high noise levels present for individual gene variants even in deeply sequenced

173 shotgun metagenomes from controlled exposure studies.

\section{The limits to quantification}

175 Noting the much more instable levels of mutations in the shotgun metagenomes, we next 176 investigated the effects of sequencing depth on the ability of our method to detect significantly

177 altered mutation frequencies. For this analysis, we used downsampled data from the shotgun

178 metagenomic library of the ciprofloxacin exposure study (Figure 2). As expected, we found that

179 the number of significantly altered mutation frequencies detected increased with larger

180 sequencing depth (Figure 2A). In addition, the average effect size of the significant mutations

181 became gradually lower with larger sequence depth, also in accordance with expectations (Figure

182 2B). Importantly, the average effect size of detectable mutation frequency differences seems to

183 decrease linearly with sequencing depth. This means that we can calculate an expected detection

184 limit for the method given the characteristics of the data and experimental setup. At 10 million

185 reads, we expect that the proportion of reads with mutation must be $30-40 \%$ higher in the

186 exposed sample in order for it to be detected as significant. The required effect decreases to, on

187 average, $10 \%$ higher at 50 million reads (Figure $2 \mathrm{~B}$ ). These numbers are of course also dependent

188 on other factors, such as the number of replicates per treatment, but nevertheless they can be

189 used as ballpark numbers to aid the design of metagenomic studies or to interpret non-significant

190 results derived from Mumame analyses. 
A)

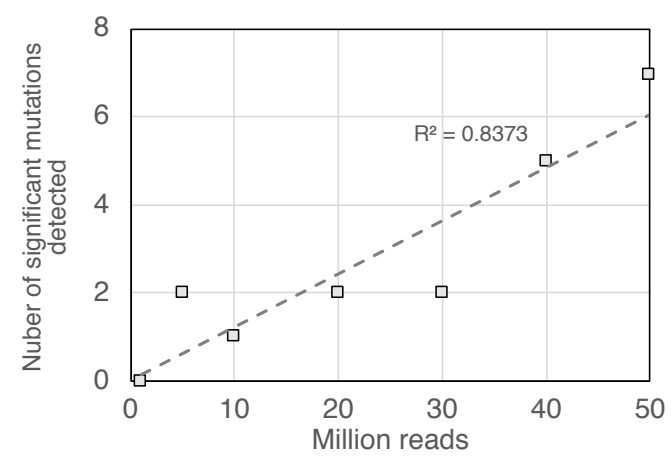

B)

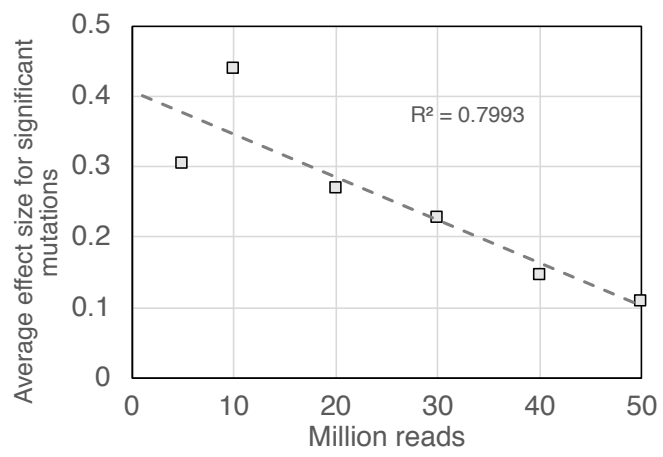

191

\section{Tetracycline-exposed Escherichia coli populations do not harbor higher abundances of resistance mutations}

After performing the validation and limitation testing of the method, we next used Mumame to quantify resistance mutations in a similar controlled aquarium setup under exposure to the antibiotic tetracycline (Lundström et al. 2016). In this study, no amplicon sequencing of the target gene for tetracycline - the $23 \mathrm{~S}$ rRNA - was performed, and thus there was no a priori true result that we could compare to. While Mumame was able to successfully detect tetracycline resistance mutations in the data, we somewhat surprisingly found no enrichment of tetracycline resistance mutations in this data (Figure 3). Notably, this result was obtained despite a very high sequencing depth (on average 181,595,072 paired-end sequences per library). Obtaining a negative result at this sequencing depth suggests that there actually is no enrichment of known E. coli resistance mutations in the samples. 


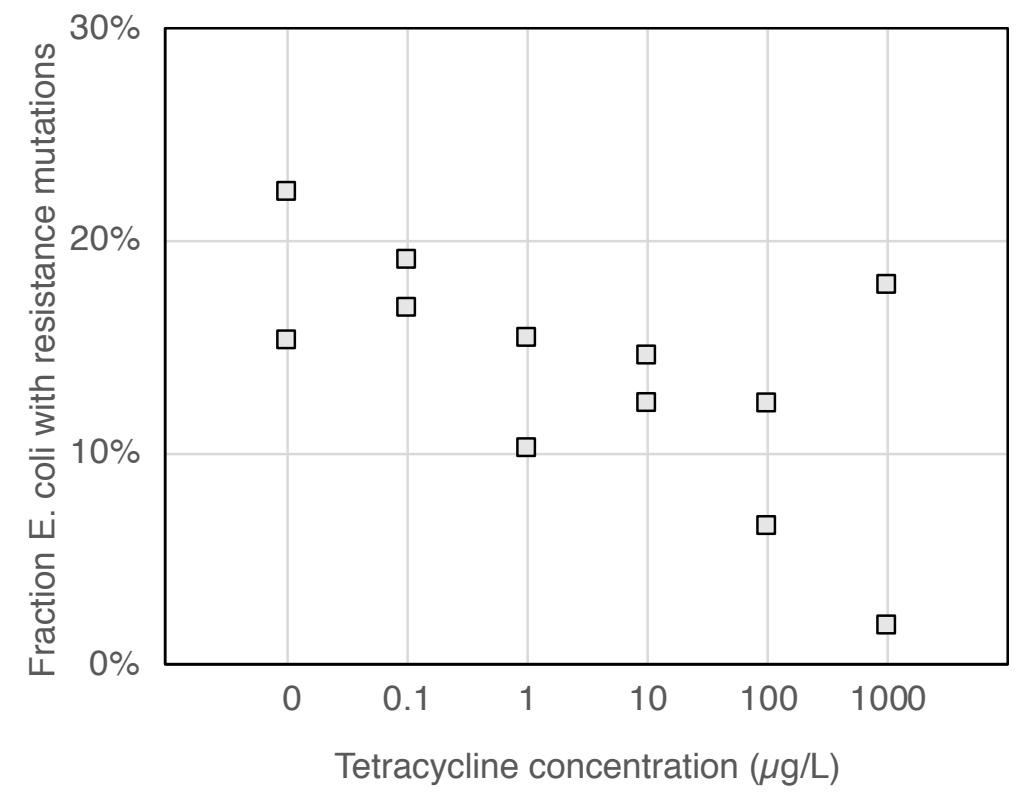

208 Figure 3. Frequencies of E. coli tetracycline resistance mutations at exposure to different concentrations of tetracycline, based on shotgun metagenomic data.

\section{Fluoroquinolone resistance mutations in ciprofloxacin-polluted sediments}

212 As a final investigation of the performance of the method, we also let Mumame quantify the

213 fluoroquinolone resistance mutations in river and lake sediments polluted by antibiotic

214 manufacturing waste, primarily ciprofloxacin (Kristiansson et al. 2011; Bengtsson-Palme et al.

215 2014; Pal et al. 2016). These libraries are fairly old and were not as deeply sequenced as the other

216 data sets we investigated. While the experimental setup of these studies in terms of number of

217 samples does not allow for proper statistical testing, we did find an enrichment of the

218 fluoroquinolone resistance mutation frequencies downstream of the pollution source, at least for

219 the E. coli gyr $A$ and $\operatorname{par} C$ genes (Figure 4). We also detected a few such mutations in other species,

220 but the counts of those were low and the results largely non-informative due to the small number

221 of detections per mutation (Figure 5).

12 


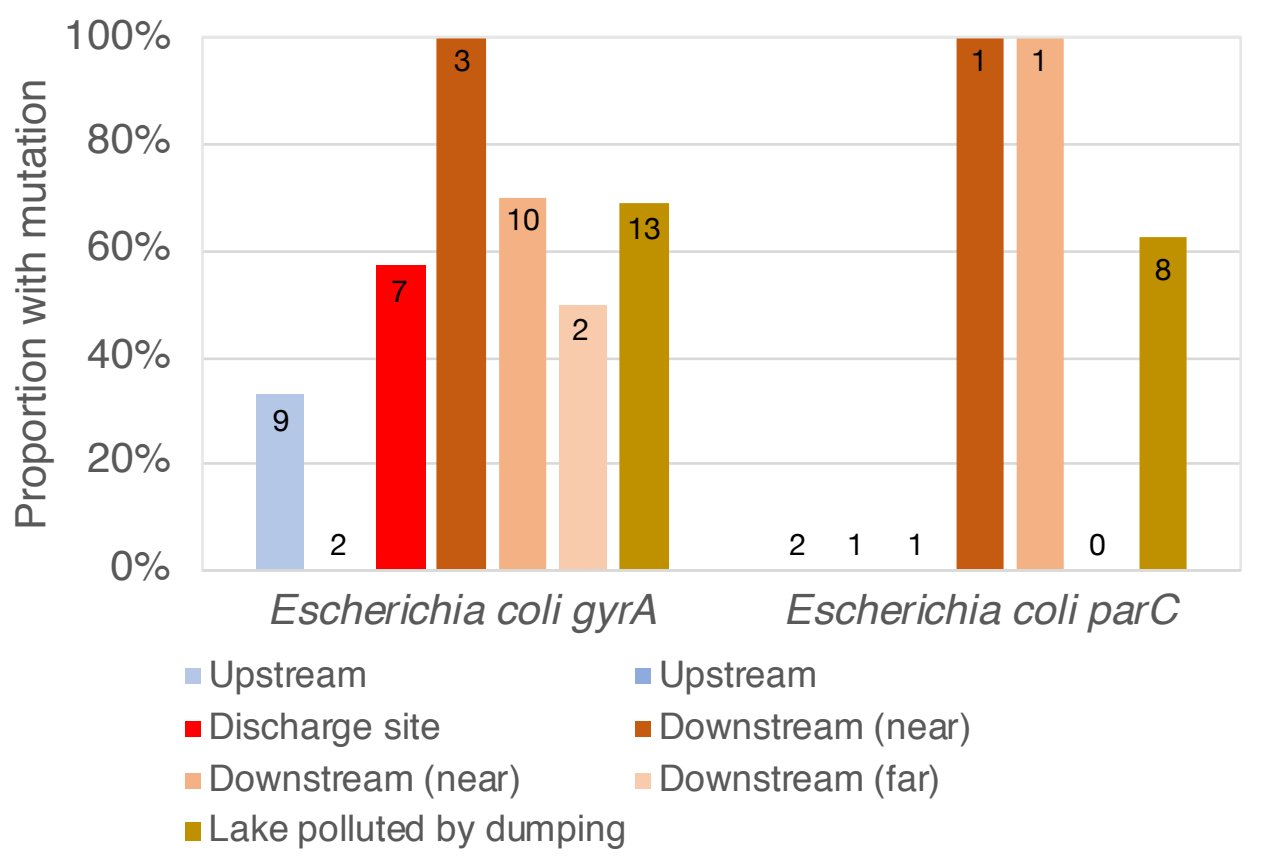

223 Figure 4. Relative frequency of $g y r A$ and $\operatorname{par} C$ sequences with resistance mutations in samples taken downstream, at or upstream of the pharmaceutical production wastewater treatment plant, as well as in a lake polluted by dumping of pharmaceutical production waste. The numbers at the top of the bars shows the total number of sequences (wildtype or mutated) identified in each sample. 
A)

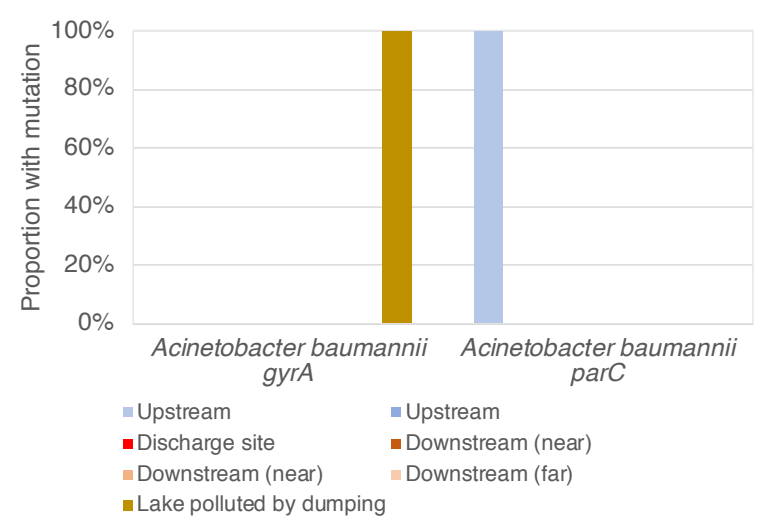

B)

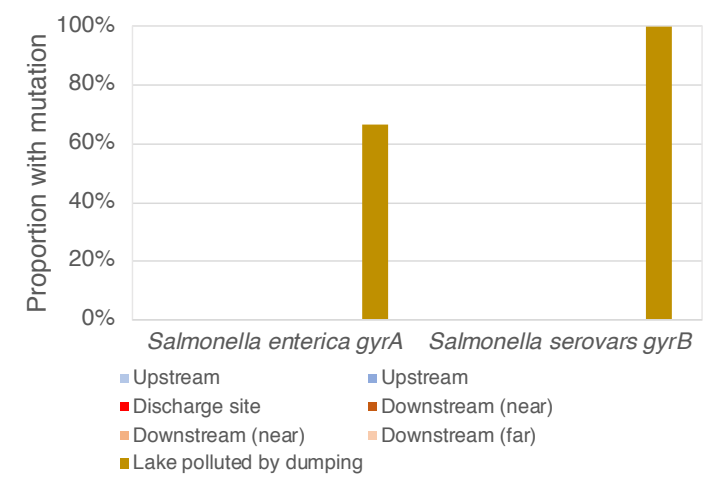

C)

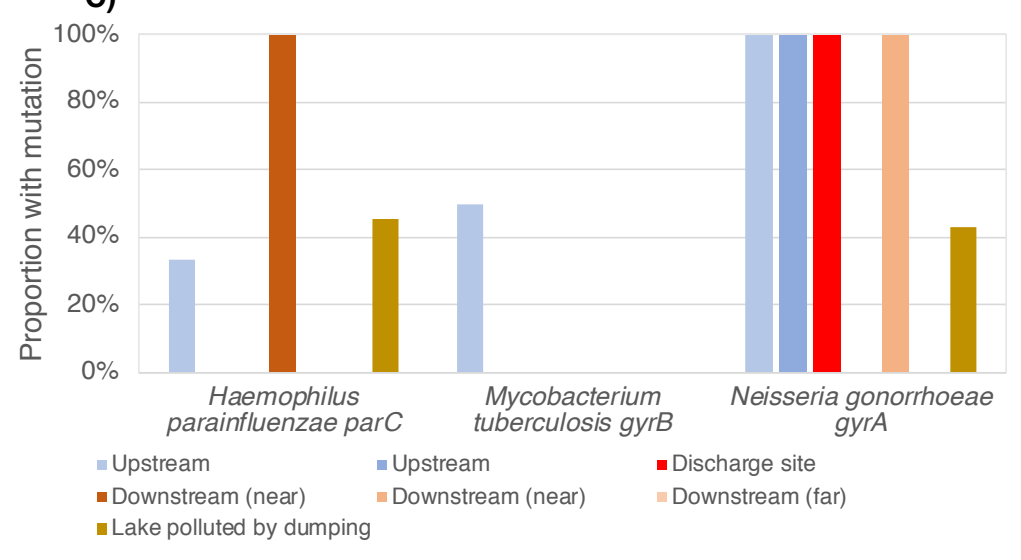

229 Figure 5. Relative frequency of sequences with resistance mutations in samples taken downstream, at or upstream of the pharmaceutical production wastewater treatment plant, as well as in a lake polluted by dumping of pharmaceutical production waste, for Acinetobacter baumanii (A), Salmonella species (B) and Haemophilus parainfluenzae, Mycobacterium tubercolosis and Neisseria gonorrhoeae (C).

\section{Discussion}

235 Metagenomics often becomes restricted to investigate gross compositional changes to the

236 taxonomy and function of microbial communities. Unfortunately, this obscures important

237 variation between individual sequence variants that may have large outcomes on phenotypes 
238 (Österlund et al. 2017; Bengtsson-Palme 2018). One example of such point mutations inducing

239 strong phenotypic changes is resistance mutations in the target genes of antibiotics (Kraupner et

240 al. 2018). However, including mutated sequence variants in the antibiotic resistance gene

241 databases is complicated, and can lead to gross misinterpretations of the data (see for example

242 (Ma et al. 2014). Still, understanding relevant variation between sequences and linking that to

243 phenotypes is somewhat of a holy Grail of metagenomics. This study has made clear that we are

244 not yet at that point in terms of bioinformatic methods and the sequencing depth required to

245 draw firm conclusions. That said, we show in this work that identifying significant and relevant

246 differences in resistance mutation frequencies between sample groups from shotgun

247 metagenomic data is possible, given a sufficiently large sequence depth. However, the

248 quantitative estimates still seem to be highly variable, even at very large sequencing depths.

249 The results of the Mumame evaluation also provides a few other important clues on potential

250 pitfalls with inferring mutation frequencies from shotgun metagenomic data. An important such

251 aspect is the disparity between mutation frequencies described by amplicon sequencing and

252 shotgun data. Particularly, the ability to relatively consistently identify the A67 and S83 mutations

253 in $\operatorname{par} C$, while the D87 mutation is seemingly less frequent in the shotgun data is somewhat

254 troubling if the goal is to identify the actual abundances of such mutations. At the same time, the

255 statistical significance of those differences could still be identified. For the A67 and S83

256 mutations, only 5 million reads were required for a significant effect to be detected, while for the

257 D87 mutations a sequencing depth of 50 million reads was required. This is not necessarily a

258 shortcoming of the Mumame software, but may just as well be due to the much noisier nature of 
In terms of interpreting the results from the exposure experiments, it is interesting to note the

counts from metagenomic sequence data compared to the large number of reads corresponding to the same genes deriving from amplicon data (Jonsson et al. 2016a).

Another important potential problem highlighted by our evaluation is the need to produce very large sequence data sets to be able to identify and quantify mutations (and wildtype) sequences with any certainty. As a rule of thumb, the targeted regions represent less than $0.004 \%$ of the bacterial genome, and each bacterial strain may correspond to only a fraction of a percent of the reads in the shotgun sequence data (depending on its abundance). This means that to identify a single read from a resistance region in the data, one would - on average - need to sequence more than five million reads. To get a reasonably confident measure of reads stemming from wildtype versus strains with mutations, approximately 10 reads from each group would be needed per sample (or, say, 20 reads in total). That would, as a rough estimate, correspond to a hundred million reads per sample. This is, unfortunately, way more sequences than what is typically generated per sample by shotgun metagenomic sequencing projects. In this study, only the samples from the tetracycline exposure study corresponded to such a high sequencing depth. Naturally, these numbers would depend on the proportions of the targeted microorganisms as well as their genome sizes, but ultimately this still presents the largest limitation to mutation studies based on metagenomic sequence data. Potentially, this problem could be partially alleviated by analyzing sufficiently large cohorts and perform the statistical analysis for general trends, but even large cohorts would be insufficient for mutations rare enough to pass below the detection limit.

overall clear increase of fluoroquinolone resistance mutations at the highest ciprofloxacin 
concentration, which nearly perfectly correspond to increases in mobile qnr fluoroquinolone genes in the same samples (Kraupner et al. 2018). This is contrasted by the trend seen in the tetracycline exposure experiments, where tetracycline resistance genes - specifically efflux pumps - were enriched at higher tetracycline concentrations (Lundström et al. 2016), while tetracycline resistance mutation abundances were not significantly altered. This non-significant result was obtained despite the exceptionally high sequencing depth of those samples.

287 While we did not have data from a proper experimental setup to address differences between 288 sediments exposed to different degrees of fluoroquinolone pollution, the quantification of resistance mutations seems to provide an important piece of information to explain the results of previous studies of resistance gene abundances in these river samples (Kristiansson et al. 2011).

291 In the original paper, the abundance of mobile fluoroquinolone resistance genes (qnr genes) were

292 shown to be enriched in the low-level polluted upstream samples, compared to the highly 293 polluted downstream samples. Importantly, the qnr genes only provide resistance to relatively low 294 levels of fluoroquinolones (Hooper and Jacoby 2015), and the authors of hypothesize that 295 chromosomal mutations of the target genes are probably necessary to survive the selection 296 pressure from antibiotics downstream of the pollution source. In this work, we show that this 297 assumption is likely correct. Only a limited number of reads were mapping to these resistance 298 regions and the number of samples unfortunately prevents us from properly assessing a statistical 299 difference between the upstream and downstream samples. Still, the proportion of resistance 300 mutations seems to be systematically higher in the samples downstream of the pollution source, 301 at least for E. coli. This indicates that the method we present here can provide important 
additional information to metagenomic studies of resistance patterns in different environment types, given that a sufficient sequencing depth is achieved.

We have here shown the utility of the Mumame tool for finding resistance mutations in shotgun metagenomic data. In this paper, we have used the CARD database (Jia et al. 2016) as the information source for resistance mutation, but the tool is flexible to use any source of such data. It is also not in any means restricted to the mutations investigated in this paper but is fundamentally agnostic to the input data. It can also be used in open screening for mutations in any gene present in the database in parallel, and can handle different mutations in both RNA and protein coding genes. The tool is flexible and fast and can therefore be implemented as a part

311 nearly any screening pipeline for antibiotic resistance data in metagenomic data sets.

\section{Conclusion}

313 This paper presents a software tool called Mumame to analyze shotgun metagenomic data for

314 point mutations, such as those conferring antibiotic resistance to bacteria. Mumame distinguish

315 between wildtype and mutated gene variants in metagenomic data and quantify them, given a

316 sufficient sequencing depth. We also provide a statistical framework for handling the generated

317 count data and account for factors such as differences in sequencing depth. Importantly, our

318 study also reveals the importance of a high sequencing depth - preferably more than 50 million

319 sequenced reads per sample - in order to get reasonably accurate estimates of mutation

320 frequencies, particularly for rare genes or species. The Mumame software package is freely

321 available from http://microbiology.se/software/mumame. We expect Mumame to be a useful 
addition to metagenomic studies of e.g. antibiotic resistance, and to increase the detail by which metagenomes can be screened for phenotypically important differences.

\section{Acknowledgements}

This work was funded by the Swedish Research Council for Environment, Agricultural Sciences and Spatial Planning (FORMAS; grant 2016-00768).

\section{Author contributions}

JBP conceived of the study. SM and JBP collected and analyzed the data. JBP designed and wrote the software package. VJ provided statistical guidance for the R implementation. JBP wrote the draft manuscript. All authors interpreted the data and contributed to the writing of the paper.

\section{Conflict of interest}

The authors have no conflicts of interest to declare.

\section{References}

Babraham Bioinformatics (2012) Trim Galore! https://www.bioinformatics.babraham.ac.uk/projects/trim_galore/

Bahram M, Hildebrand F, Forslund SK, et al (2018) Structure and function of the global topsoil microbiome. Nature 320:1039. doi: 10.1038/s41586-018-0386-6 


\section{Bengtsson-Palme J (2018) Strategies for Taxonomic and Functional Annotation of} Metagenomes. In: Nagarajan M (ed) Metagenomics: Perspectives, Methods, and Applications. Academic Press, Elsevier, Oxford, UK,

Bengtsson-Palme J, Boulund F, Fick J, et al (2014) Shotgun metagenomics reveals a wide array of antibiotic resistance genes and mobile elements in a polluted lake in India. Front Microbiol 5:648. doi: 10.3389/fmicb.2014.00648

Bengtsson-Palme J, Larsson DGJ, Kristiansson E (2017) Using metagenomics to investigate human and environmental resistomes. Journal of Antimicrobial Chemotherapy 72:26902703. doi: $10.1093 / \mathrm{jac} / \mathrm{dkx} 199$

Edgar RC (2010) Search and clustering orders of magnitude faster than BLAST. Bioinformatics 26:2460-2461. doi: 10.1093/bioinformatics/btq461

Hooper DC, Jacoby GA (2015) Mechanisms of drug resistance: quinolone resistance. Ann N Y Acad Sci 1354:12-31. doi: 10.1111/nyas.12830

Human Microbiome Project Consortium (2012) Structure, function and diversity of the healthy human microbiome. Nature 486:207-214. doi: 10.1038/nature11234

Jia B, Raphenya AR, Alcock B, et al (2016) CARD 2017: expansion and model-centric curation of the comprehensive antibiotic resistance database. Nucleic Acids Res gkw1004. doi: $10.1093 / \mathrm{nar} / \mathrm{gkw} 1004$

Johnning A, Kristiansson E, Angelin M, et al (2015a) Quinolone resistance mutations in the faecal microbiota of Swedish travellers to India. BMC Microbiol 15:235. doi: 10.1186/s12866-015-0574-6

Johnning A, Kristiansson E, Fick J, et al (2015b) Resistance Mutations in gyrA and parC are Common in Escherichia Communities of both Fluoroquinolone-Polluted and Uncontaminated Aquatic Environments. Front Microbiol 6:1355. doi: 10.3389/fmicb.2015.01355

Johnning A, Moore ERB, Svensson-Stadler L, et al (2013) Acquired genetic mechanisms of a multiresistant bacterium isolated from a treatment plant receiving wastewater from antibiotic production. Appl Environ Microbiol 79:7256-7263. doi: 10.1128/AEM.02141-13

Jonsson V, Österlund T, Nerman O, Kristiansson E (2016a) Variability in Metagenomic Count Data and Its Influence on the Identification of Differentially Abundant Genes. Journal of Computational Biology cmb.2016.0180. doi: 10.1089/cmb.2016.0180

Jonsson V, Österlund T, Nerman O, Kristiansson E (2016b) Statistical evaluation of methods for identification of differentially abundant genes in comparative metagenomics. BMC Genomics 17:78. doi: 10.1186/s12864-016-2386-y 
Kraupner N, Ebmeyer S, Bengtsson-Palme J, et al (2018) Selective concentration for ciprofloxacin resistance in Escherichia coli grown in complex aquatic bacterial biofilms. Environ Int 116:255-268. doi: 10.1016/j.envint.2018.04.029

Kristiansson E, Fick J, Janzon A, et al (2011) Pyrosequencing of antibiotic-contaminated river sediments reveals high levels of resistance and gene transfer elements. 6:e17038. doi: 10.1371/journal.pone.0017038

Lundström SV, Östman M, Bengtsson-Palme J, et al (2016) Minimal selective concentrations of tetracycline in complex aquatic bacterial biofilms. Sci Total Environ 553:587-595. doi: 10.1016/j.scitotenv.2016.02.103

Ma L, Li B, Zhang T (2014) Abundant rifampin resistance genes and significant correlations of antibiotic resistance genes and plasmids in various environments revealed by metagenomic analysis. Appl Microbiol Biotechnol 98:5195-5204. doi: 10.1007/s00253-014-5511-3

Österlund T, Jonsson V, Kristiansson E (2017) HirBin: high-resolution identification of differentially abundant functions in metagenomes. BMC Genomics 18:316. doi: 10.1186/s12864-017-3686-6

Pal C, Bengtsson-Palme J, Kristiansson E, Larsson DGJ (2016) The structure and diversity of human, animal and environmental resistomes. Microbiome 4:54. doi: 10.1186/s40168-0160199-5

Quince C, Walker AW, Simpson JT, et al (2017) Shotgun metagenomics, from sampling to analysis. Nat Biotechnol 35:833-844. doi: 10.1038/nbt.3935

R Core Team (2016) R: A Language and Environment for Statistical Computing. R Foundation for Statistical Computing, Vienna, Austria

Sunagawa S, Coelho LP, Chaffron S, et al (2015) Ocean plankton. Structure and function of the global ocean microbiome. Science 348:1261359. doi: 10.1126/science.1261359

Yooseph S, Sutton G, Rusch DB, et al (2007) The Sorcerer II Global Ocean Sampling expedition: expanding the universe of protein families. 5:e16. doi: 10.1371/journal.pbio.0050016 